\title{
Enhanced recovery pathways in thoracic surgery from Italian VATS Group: perioperative analgesia protocols
}

\author{
Federico Piccioni ${ }^{1}$, Matteo Segat ${ }^{2}$, Stefano Falini ${ }^{2}$, Marzia Umari ${ }^{2}$, Olga Putina ${ }^{3}$, Lucio Cavaliere ${ }^{4}$, \\ Riccardo Ragazzi ${ }^{5}$, Domenico Massullo ${ }^{6}$, Marco Taurchini ${ }^{7}$, Carlo Del Naja ${ }^{7}$, Andrea Droghetti ${ }^{8}$ \\ ${ }^{1}$ Department of Critical Care Medicine and Support Therapy, Fondazione IRCCS Istituto Nazionale dei Tumori, Milan, Italy; ${ }^{2}$ Department of \\ Perioperative Medicine, Intensive Care and Emergency, Cattinara University Hospital, Trieste, Italy; ${ }^{3}$ Anesthesiology and Intensive Care Unit, \\ ASST, Mantova, Italy; ${ }^{4}$ Anesthesiology and Intensive Care Unit I, IRCCS Casa Sollievo della Sofferenza Hospital, San Giovanni Rotondo (FG), Italy; \\ ${ }^{5}$ Department of Morphology, Surgery and Experimental Medicine, Azienda Ospedaliero-Universitaria Sant'Anna, Ferrara, Italy; ${ }^{6}$ Anesthesiology \\ and Intensive Care Unit, Azienda Ospedaliera Sant'Andrea, Rome, Italy; ${ }^{7}$ Department of Thoracic Surgery, IRCCS Casa Sollievo della Sofferenza \\ Hospital, San Giovanni Rotondo (FG), Italy; ${ }^{8}$ Thoracic Surgery Division, ASST, Mantova, Italy \\ Contributions: (I) Conception and design: F Piccioni, M Umari, O Putina, M Taurchini, A Droghetti; (II) Administrative support: R Ragazzi, \\ D Massullo; (III) Provision of study materials or patients: O Putina, L Cavaliere, C Del Naja; (IV) Collection and assembly of data: F Piccioni, \\ M Segat, S Falini; (V) Data analysis and interpretation: F Piccioni, M Umari, O Putina, L Cavaliere; (VI) Manuscript writing: All authors; (VII) Final \\ approval of manuscript: All authors. \\ Correspondence to: Carlo Del Naja, MD. Casa Sollievo della Sofferenza Hospital, viale Cappuccini, 1, 71013 San Giovanni Rotondo (FG), Italy. \\ Email: c.delnaja@operapadrepio.it.
}

\begin{abstract}
Video-assisted thoracoscopic surgery (VATS) is a minimally invasive technique that allows a faster recovery after thoracic surgery. Although enhanced recovery after surgery (ERAS) principles seem reasonably applicable to thoracic surgery, there is little literature on the application of such a strategy in this context. In regard to pain management, ERAS pathways promote the adoption of a multimodal strategy, tailored to the patients. This approach is based on combining systemic and loco-regional analgesia to favour opioid-sparing strategies. Thoracic paravertebral block is considered the first-line loco-regional technique for VATS. Other techniques include intercostal nerve block and serratus anterior plane block. Nonsteroidal anti-inflammatory drugs and paracetamol are essential part of the multimodal treatment of pain. Also, adjuvant drugs can be useful as opioid-sparing agents. Nevertheless, the treatment of postoperative pain must take into account opioid agents too, if necessary. All above is useful for careful planning and execution of a multimodal analgesic treatment to enhance the recovery of patients. This article summarizes the most recent evidences from literature and authors' experiences on perioperative multimodal analgesia principles for implementing an ERAS program after VATS lobectomy.
\end{abstract}

Keywords: Regional analgesia; pain management; video-assisted thoracoscopic surgery (VATS)

Submitted Nov 16, 2017. Accepted for publication Dec 11, 2017.

doi: $10.21037 /$ jtd.2017.12.86

View this article at: http://dx.doi.org/10.21037/jtd.2017.12.86

\section{Introduction}

Enhanced Recovery After Surgery (ERAS) is a multimodal, multidisciplinary approach to surgical patients with the aim of enhancing the quality of recovery after surgery $(1,2)$. This strategy translates into faster post-operative recovery and improvements of outcomes. All the ERAS Society guidelines (freely available at www.erassociety.org) take into consideration the perioperative management of analgesia. The role of pain management in ERAS pathways is fundamental, considering the importance of containing surgical stress, reducing pain-related complications and speeding recovery (2-5). 
Although the ERAS principles seem reasonably applicable to thoracic surgery, there is little literature on the application of such a strategy in this context. For certain, the evolution of minimally invasive thoracic surgery has created a more favourable framework for the drafting of ERAS programs. In regard to pain management, it promotes the adoption of a multimodal strategy, tailored to the patients. Yet there is currently no consensus on the best strategy for treating pain after video-assisted thoracoscopic surgery (VATS) (6). Despite the reduced invasiveness when compared to the thoracotomic approach, postoperative pain after VATS must still be considered moderate-severe $(7,8)$.

This article summarizes the most recent evidences from literature and authors' experiences on perioperative multimodal analgesia principles for implementing an ERAS program after VATS lobectomy. Some technical or pharmacological aspects of pain therapy and postoperative nausea and vomit prevention are not herein discussed because already analyzed in other articles included in this issue of the journal.

\section{Multimodal analgesia strategy}

Multimodal analgesia is the most effective strategy to improve pain relief and reduce the side effects of every single agent $(4,9,10)$. It is based on the use of a variety of analgesic agents and/or techniques that target different nociceptive mechanisms. The latest comprehensive guidelines on postoperative analgesia, published in 2016 by the American Pain Society, the American Society of Regional Anesthesia and Pain Medicine, and the American Society of Anesthesiologists' Committee on Regional Anesthesia, strongly recommends that clinicians offer multimodal analgesia for the treatment of postoperative pain in both children and adults (strong recommendation, high-quality evidence) (9). Back in 2010, the Italian Society of Anesthesia, Analgesia and Intensive Care (SIAARTI) already recommended the adoption of multimodal analgesic strategies and underlined the importance of their adjustment to fast-track surgical settings (10). Recent reviews on analgesic care in ERAS protocols report the same concept, highlighting the necessity of combining systemic and loco-regional analgesia to favour opioidsparing strategies $(4,5,11)$. The latter goal is extremely important, to minimize not only the side effects of this pharmacological class, but also the risk of respiratory complications of patients undergoing thoracic surgery.

\section{Systemic intravenous analgesia}

Systemic analgesia is a fundamental component of any multimodal approach. The administration of analgesic drugs must be scheduled and include a rescue strategy for inadequate pain relief (10).

\section{Opioids}

Opioids have largely been used for pain management after thoracic surgery because of its severity. Pre-emptive administration of these agents is no longer recommended $(9,12)$. Given the wide interindividual response, metabolism and elimination of opioids, their administration should be delivered via patient-controlled analgesia (PCA) devices (9) avoiding intravenous continuous infusion or intramuscular administration of opioids. Obviously, impaired cognitive function contraindicates PCA use (9). Finally, routine basal infusion of opioids with PCA must be avoided in opioidnaive patients because it increases the risk of side effects (9,13-16). Opioid-related side effects are in fact numerous, and include hypotension, respiratory depression, itching, nausea and vomit, bowel ileus, confusion and sedation $(16,17)$. For these reasons, whenever possible, the use of opioids should be avoided or at least reduced to enhance recovery after VATS lobectomy. However, opioids still represent a significant component of pain therapy, and can be administered as a rescue analgesic when other nonopioid analgesics fail.

Morphine is the most used opioid for pain management after surgery. It has an onset time of about 30 minutes and a duration of action of 4-6 h after intravenous administration $(18,19)$. Some of its metabolites are actually more active than morphine itself and can accumulate in case of kidney failure (18), so its clinical effect must be carefully monitored. During PCA administration, the typical dose is $1-2 \mathrm{mg}$ bolus with a lock-out interval of 6-12 minutes $(15,16,20)$.

Fentanyl and sufentanil are powerful lipophilic opioids with faster onset time and shorter duration of action when compared with morphine. Fentanyl is frequently used as the intraoperative opioid of choice. Both fentanyl and sufentanil can technically be used for PCA, but no robust data is available on their use for acute pain management 
after thoracic surgery. Conversely, their use is common in combination with local anesthetics as part of epidural analgesia. Recently, a sufentanil sublingual tablet system has been proposed as a novel PCA device $(21,22)$. It delivers $15 \mathrm{mcg}$ of sufentanil with a lock-out interval of 20 minutes. This non-invasive PCA system is attractive and potentially adapt to an ERAS program but no data is currently available in regard to thoracic surgery.

Oxycodone is another opioid administered via the oral route with an onset time of 1-2 h and a duration of action of $4 \mathrm{~h}$. Its use has been successfully proposed for postoperative pain management of patients undergoing thoracoscopic surgery once they have resumed oral intake (23).

Given the reduced invasiveness of VATS procedures, weak opioids are suitable for postoperative acute pain, if associated with a proper loco-regional technique. Codeine is extensively used for the treatment of moderate pain. It is usually administered via oral route combined with paracetamol to exploit their synergistic effect $(24,25)$. Tramadol can instead be used intravenously in the immediate postoperative period. It produces analgesia through two mechanisms of action: mu-opioid receptor activation and serotonin and norepinephrine reuptake inhibition. It must be noticed that the concomitant use of ondansetron, a commonly associated antiemetic agent, can reduce its efficacy due to its effect on serotonin receptors (26). Tramadol is usually administered intravenously (100 mg every 6-8 h) or orally (50-100 $\mathrm{mg}$ every $6-8 \mathrm{~h}$ ) for the treatment of mild and moderate pain, and is also useful for the prosecution of an analgesic therapy based on a strong opioid (i.e., morphine).

\section{Nonsteroidal anti-inflammatory drugs (NSAIDs)}

NSAIDs administration reduces opioids demand of $30-35 \%$ (27). They increase the risk of kidney failure, gastric bleeding and have antiaggregant platelets effect. Recently, a meta-analysis has denied its role in increasing bleeding risk after surgery (28). There are no specific data on this topic as regard thoracic surgery but there is no reason to hypothesize the contrary. Nowadays, ketorolac is the most used NSAID in the postoperative phase. Its posology is $30 \mathrm{mg}$ i.v. every $8 \mathrm{~h}$ for 2 days (29). Ketorolac and diclofenac have both shown efficacy after VATS, with comparable results (30). Indomethacin has been associated with a better pain relief after thoracotomy (31) but its use is uncommon in this context.

\section{Paracetamol}

Like NSAIDs, paracetamol enhances analgesia and produces an opioid-sparing effect, reducing postoperative postoperative nausea and vomiting (PONV) and sedation (32-35). It is well tolerated and not burdened by NSAID-like side effects. Its only limit is the potential for hepatotoxicity but at doses of more than $4 \mathrm{~g}$ per day. Moreover, a randomized controlled trial (RCT) showed a decrease of postoperative ipsilateral shoulder pain after thoracotomy (36). The intravenous dosage for postoperative pain is $1 \mathrm{~g}$ every 6-8 h. Given its safe pharmacodynamic profile and its synergic effect with other analgesics, paracetamol must be considered as a first choice in a multimodal analgesic protocol.

\section{N-methyl-D-aspartate (NMDA) antagonists}

The inhibition of NMDA receptors decreases postoperative pain and opioid consumption (9). Ketamine is used at subanesthetic doses as an infusion during and after surgery. It can reduce intravenous PCA morphine use and PONV (37). Although it happens rarely, one must consider that it can cause dysphoria. Magnesium sulphate is another NMDA antagonist that can decrease postoperative pain and opioid consumption (38).

\section{Dexamethasone}

Dexamethasone is a steroid agent useful for PONV prevention (10). A recent meta-analysis demonstrated that it also decreases postoperative pain and opioid consumption (39). Particularly, the 8-mg dose does not seem to be superior than the $4 \mathrm{mg}$ one.

\section{Lidocaine}

Lidocaine hydrochloride is a short acting local anesthetic that can be administered as an intravenous infusion during and after surgery. Its use is implemented in many ERAS program (5). The infusion of lidocaine for thoracotomy has resulted in reduced pain and morphine consumption in the 
first $6 \mathrm{~h}$ after intervention (40). Unfortunately, this result was not confirmed after VATS surgery (41). Given the lack of evidence of lidocaine's efficacy in this context, it seems reasonable to consider it as a second line treatment.

\section{Gabapentinoids}

Gabapentinoids (gabapentin and pregabalin) are antiepileptic agents commonly used to treat neuropathic pain. Their use has been proposed as opioid-sparing agents and to prevent the insurgence of post-thoracotomy neuropathic pain. Meta-analyses indicate that preoperative administration of gabapentinoids may also reduce postoperative pain and opioid consumption $(42,43)$. Preoperative gabapentin before thoracotomy has not shown to reduce pain, opioid consumption nor the prevalence of neuropathic pain at 3 months after surgery (44). Conversely, pregabalin has demonstrated promising results in terms of post-thoracotomy neuropathic pain $(45,46)$. There is no data concerning the use of gabapentinoids in the context of VATS surgery. Both agents (especially gabapentin) are associated with sedation, dizziness, and visual disturbances. Considering these side effects, gabapentinoids must be used with caution and cannot be recommended for routine use in ERAS protocols, especially in elderly patients.

\section{Loco-regional analgesia}

\section{Thoracic epidural analgesia (TEA)}

TEA is considered the gold standard technique for pain management after thoracic surgery $(47,48)$ and recommended as first choice in many ERAS protocols for other surgeries (especially for open surgical cases) $(4,5)$. It provides a better pain relief than opioid PCA treatment and promotes recovery. Opioids can be associated to local anesthetics to increase the efficacy of epidural analgesia. Along with TEA's effectiveness, its invasiveness must be taken into account, which might limit its use in some circumstances (i.e., use of anticoagulants). TEA has several other limitations: the placement of thoracic epidural catheter can be challenging, there is a need for adequate skilled care providers for its perioperative management, and it can cause sympathetic blockade, respiratory depression, urinary retention and, rarely, local complications both during and after procedure. Today, the most used local anesthetics for postoperative TEA are ropivacaine $0.2 \%$ and levobupivacaine $0.125 \%$ at an infusion rate usually between $6-10 \mathrm{~mL} / \mathrm{h}$.

\section{Thoracic paravertebral block (TPVB)}

In the context of mini-invasive surgical approaches, such as VATS lobectomy, it becomes imperative to evaluate the risk-benefit ratio of any invasive loco-regional technique. In the last 15 years, the TPVB has grown in popularity and it is now increasingly proposed as an alternative to TEA. It is comparable to TEA, but associated with less side effects (49) and an improved safety profile (50). A recent meta-analysis has equalized it to TEA with respect to pain relief, major complications, length of hospital stay and 30-day mortality for patients undergoing thoracotomy (49).

The single-shot TPVB was effective for pain control after VATS mainly in the immediate postoperative period in several studies (51). For an adequate postoperative pain management, it needs to be associated with an opioid-based PCA, as commonly done in UK (52). Alternatively, a longer analgesic coverage can be obtained with the continuous TPVB, i.e., the infusion of local anesthetics via a catheter placed in the paravertebral space (53).

The TPVB can be performed with the blind technique, the ultrasound-guided approach or intraoperatively by the surgeon. Currently, no study has compared these techniques but the ultrasound-guided one is reasonably the most accurate and safe approach. The TPVB is easier and safer than TEA to perform, but it is not devoid of possible complications such as pneumothorax, hemodynamic compromise, or total spinal anesthesia (54-58). Thus, new approaches to the TPVB for breast surgery have been proposed: the retrolaminar block (RLB) and the midpoint transverse process to pleura block (MTP block) $(59,60)$. In both cases, the local anesthetic is injected near the paravertebral space providing similar effect. A clear limitation of these techniques appears the inability to place a catheter for continuous postoperative infusion. At the current time, we do not recommend RLB and MTP blocks for pain management after VATS.

The best local anesthetic concentration for TPVB is not known, it ranges between bupivacaine $0.1-0.25 \%$ at $5-12 \mathrm{~mL} / \mathrm{h}$ to ropivacaine $0.2 \%$ at $4 \mathrm{~mL} / \mathrm{h}$ (18). The addition of clonidine or fentanyl to the TPVB has not shown benefit after thoracotomy (61) and is not recommended. 


\section{Intercostal nerve block (ICNB)}

ICNB is a well-known, fairly simple technique for pain management after thoracotomy (47). Both the single-shot technique and the continuous infusion are possible, but only the latter seems effective after thoracic surgery (62). Other successful strategies contemplate the use of liposomal formulations $(63,64)$. The continuous infusion of local anesthetic in the intercostal space provides adequate pain relief, comparable to TEA, until the 5th postoperative days after thoracotomy (65). After VATS, it is effective for up to $16 \mathrm{~h}$ and reduces morphine consumption for the first $24 \mathrm{~h} \mathrm{(53).}$ In conclusion, ICNB is suitable in combination with PCA therapy when TEA or TPVB have not been performed (47).

\section{Serratus anterior plane block (SAPB)}

The SAPB was originally proposed for breast surgery (66) but its applications have later been extended, and is now often used in thoracic surgery. It is an ultrasound-guided thoracic wall nerve block that covers the lateral cutaneous branch of the intercostal nerves from T2 to T9 (67). The SAPB provides more hemodynamic stability compared with TEA after thoracotomy (68) and potentiates PCA analgesia reducing pain and morphine consumption (69). In the first study, a loading dose of levobupivacaine $0.25 \%$ $30 \mathrm{~mL}$ was followed by a continuous infusion at $5 \mathrm{~mL} / \mathrm{h}$ (with levobupivacaine $0.125 \%$ ). Furthermore, in a small randomized trial comparing tramadol-based PCA $v s$. tramadol-based PCA plus single-shot SAPB (bupivacaine $0.25 \% 20 \mathrm{~mL}$ ) in patients undergoing VATS, the locoregional technique reduced pain and opioid consumption in the first $24 \mathrm{~h}$ after intervention (70). The limited invasiveness of this technique is intriguing for VATS but, despite the promising results, more studies are needed to confirm its effective potential in this context. However, the $\mathrm{SAPB}$, along with the ICNB, can be considered as a second choice whenever the TPVB is not feasible. It can also be implemented before the surgical incision, and associated with the TPVB performed intraoperatively by the surgeon under direct view.

\section{Erector spinae plane block (ESPB)}

The ESPB is a newly described technique for providing thoracic anesthesia (71). Under ultrasound guidance, about $20 \mathrm{~mL}$ of local anesthetic are injected $3 \mathrm{~cm}$ beside the interspinous line at T5 level deep to the trapezius, rhomboid and erector spinae muscles. ESPB has been proposed for thoracic neuropathic pain, rib fractures and as rescue in thoracotomy after epidural failure (72-74). Currently, the lack of experience with this technique does not allow to define its role in the treatment of pain after VATS and hence its use is not yet recommended.

\section{From acute to chronic pain}

Chronic postsurgical pain is a common and serious complication after thoracic surgery [post-thoracotomy pain syndrome (PTPS)] (50,75). Risk factors for the PTPS include female gender, young age, anxiety, and depression. Its prevalence is extremely variable, ranging from $9 \%$ to $80 \%$ after thoracotomy and from $5 \%$ to $33 \%$ after VATS (76). This difference between different surgical approaches is not widely accepted (77). Intraoperative ketamine has not demonstrated any protective effect over PTPS (17). Similarly, there is no conclusive data in regard to gabapentinoids for the prevention of chronic postsurgical pain, even though pregabalin has shown promising results after thoracotomy $(48,49)$.

Today, an adequate postoperative pain relief together with the inhibition of afferent pain transmission is considered the best way to prevent PTPS $(50,78,79)$. From this point of view, loco-regional techniques play a primary role and must be considered an indispensable part of the multimodal pain therapy.

\section{Conclusions}

Effective perioperative pain management is a crucial part of any ERAS program. Careful planning and execution of the analgesic treatment, based on a multimodal approach, is mandatory to enhance the recovery of patients (Figure 1). The wide range of loco-regional analgesia techniques for thoracic surgery contributes to achieve this goal. So, we strongly suggest that all patients receive the administration of a local anesthetic through one of the techniques discussed in this article as an integral part of the VATS surgery's analgesic protocol. 


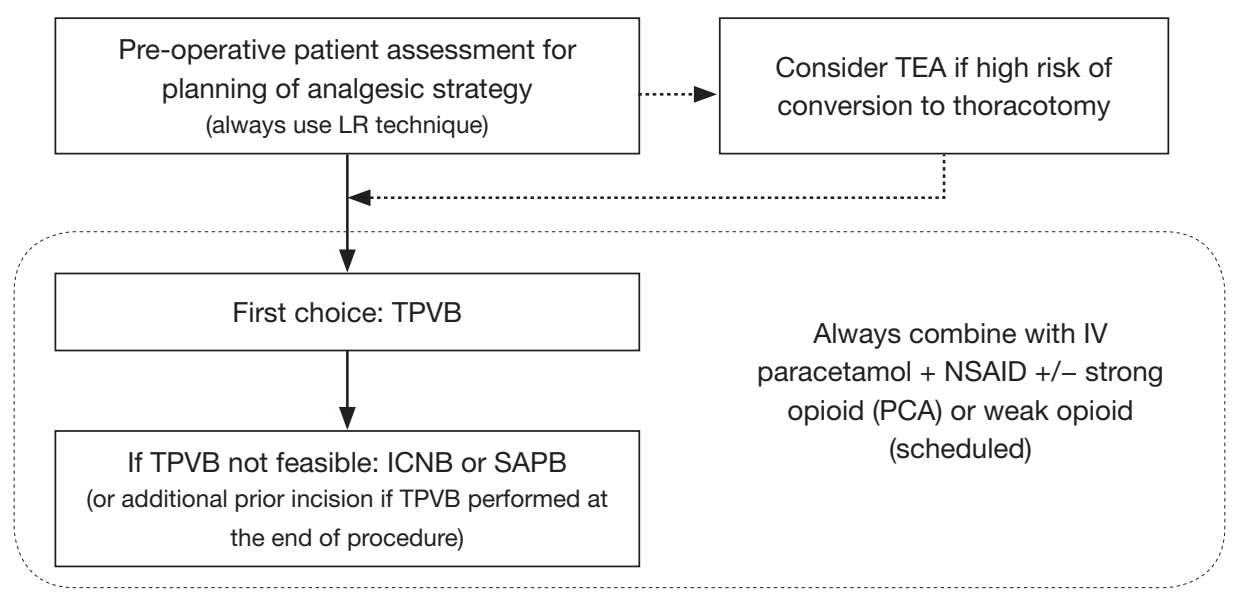

Figure 1 Schematic representation of post-operative pain management strategy after VATS lobectomy. The pre-operative patient assessment allows to set the analgesic plan. The cornerstone is always the inclusion of a loco-regional technique. Thoracic epidural analgesia should be considered if there is a high risk of conversion to thoracotomy. Otherwise thoracic paravertebral block is considered the first-line loco-regional technique. If not feasible, it can be replaced with intercostal nerve block or serratus anterior plane block. These techniques must be always integrated with systemic analgesia to meet the criteria of multimodal analgesia. LR, loco-regional; TEA, thoracic epidural analgesia; TPVB, thoracic paravertebral block; ICNB, intercostal nerve block; SAPB, serratus anterior plane block; NSAID, nonsteroidal anti-inflammatory drug; PCA, patient-controlled analgesia; VATS, video-assisted thoracoscopic surgery.

\section{Acknowledgements}

None.

\section{Footnote}

Conflicts of Interest: The authors have no conflicts of interest to declare.

\section{References}

1. Fearon KC, Ljungqvist $\mathrm{O}$, von Meyenfeldt $M$, et al. Enhanced recovery after surgery: a consensus review of clinical care for patients undergoing colonic resection. Clin Nutr 2005;24:466-77.

2. Ljungqvist O, Scott M, Fearon KC. Enhanced recovery after surgery: a review. JAMA Surg 2017;152:292-8.

3. Kehlet H, Wilmore DW. Evidence-based surgical care and the evolution of Fast-track surgery. Ann Surg 2008;248:189-98.

4. Wick EC, Grant MC, Wu CL. Postoperative multimodal analgesia pain management with nonopioid analgesics and techniques: a review. JAMA Surg 2017:152:691-7.

5. Beverly A, Kaye AD, Ljungqvist O, et al. Essential elements of multimodal analgesia in enhanced recovery after surgery (ERAS) Guidelines. Anesthesiol Clin 2017;35:e115-43.
6. Holbek BL, Horsleben Petersen R, Kehlet H, et al. Fast-track video-assisted thoracoscopic surgery: future challenges. Scand Cardiovasc J 2016;50:78-82.

7. Steinthorsdottir KJ, Wildgaard L, Hansen HJ, et al. Regional analgesia for video-assisted thoracic surgery: a systematic review. Eur J Cardiothorac Surg 2014;45:959-66.

8. Khoshbin E, Al-Jilaihawi AN, Scott NB, et al. An audit of pain control path- ways following video-assisted thoracoscopic surgery. Innovations 2011;6:248-52.

9. Chou R, Gordon DB, de Leon-Casasola OA, et al. Management of Postoperative Pain: A Clinical Practice Guideline From the American Pain Society, the American Society of Regional Anesthesia and Pain Medicine, and the American Society of Anesthesiologists' Committee on Regional Anesthesia, Executive Committee, and Administrative Council. J Pain 2016;17:131-57. Erratum in: J Pain 2016;17:508-10.

10. Savoia G, Alampi D, Amantea B, et al. Postoperative pain treatment SIAARTI recommendations 2010: short version. Minerva Anestesiol 2010:76:657-67.

11. Kumar K, Kirksey MA, Duong S, et al. A review of opioidsparing modalities in perioperative pain management: methods to decrease opioid use postoperatively. Anesth Analg 2017;125:1749-60.

12. Ong CK-S, Lirk P, Seymour RA, et al. The efficacy of preemptive analgesia for acute postoperative 
pain management: a meta-analysis. Anesth Analg 2005;100:757-73.

13. Parker RK, Holtmann B, White PF. Patient-Controlled Analgesia: Does a Concurrent Opioid Infusion Improve Pain Management After Surgery? JAMA 1991;266:1947-52.

14. Russell AW, Owen H, Ilsley AH, et al. Background infusion with patient-controlled analgesia: effect on postoperative oxyhaemoglobin saturation and pain control. Anaesth Intensive Care 1993;21:174-9.

15. Grass JA. Patient-controlled analgesia. Anesth Analg 2005;101:S44-61.

16. Palmer PP, Miller RD. Current and developing methods of patient- controlled analgesia. Anesthesiol Clin 2010;28:587-99.

17. Kolettas A, Lazaridis G, Baka S, et al. Postoperative pain management. J Thorac Dis 2015;7:S62-72.

18. Stoelting RK, Hillier SC. Pharmacology and Physiology in Anesthetic Practice. 4th edition. Lippincott Williams \& Wilkins, 2012.

19. Schug SA, Palmer GM, Scott DA, et al. Acute pain management: scientific evidence, fourth edition, 2015. Med J Aust 2016;204:315-7.

20. Badner NH, Doyle JA, Smith MH, et al. Effect of varying intravenous Patient-controlled analgesia dose and lockout interval while maintaining a constant hourly maximum dose. J Clin Anesth 1996;8:382-5.

21. Minkowitz HS, Singla NK, Evashenk MA, et al. Pharmacokinetics of sublingual sufentanil ta- blets and efficacy and safety in the management of postoperative pain. Reg Anesth Pain Med 2013;38:131-9.

22. Porela-Tiihonen S, Kokki M, Kokki H. Sufentanil sublingual formulation for the treatment of acute, moderate to severe postoperative pain in adult patients. Expert Rev Neurother 2017;17:101-11.

23. Kampe S, Weinreich G, Darr C, et al. Controlled-Release Oxycodone as "Gold Standard" for Postoperative Pain Therapy in Patients Undergoing Video-Assisted Thoracic Surgery or Thoracoscopy: A Retrospective Evaluation of 788 Cases. Thorac Cardiovasc Surg 2015;63:510-3.

24. Kress HG, Untersteiner G. Clinical update on benefit versus risks of oral paracetamol alone or with codeine: still a good option? Curr Med Res Opin 2017;33:289-304.

25. Toms L, Derry S, Moore RA, et al. Single dose oral paracetamol (acetaminophen) with codeine for postoperative pain in adults. Cochrane Database Syst Rev 2009;(1):CD001547.

26. Arcioni R, Della Rocca M, Romanò S, et al. Ondansetron inhibits the analgesic effects of tramadol: a possible
5-HT(3) spinal receptor involvement in acute pain in humans. Anesth Analg 2002;94:1553-7.

27. Boussofara M, Mtaallah MH, Bracco D, et al. Co-analgesic effect of ketoro- lac after thoracic surgery. Tunis Med 2006;84:427-31.

28. Gobble RM, Hoang HLT, Kachniarz B, et al. Ketorolac does not increase perioperative bleeding: a meta-analysis of randomized controlled trials. Plast Reconstr Surg 2014;133:741-55.

29. Laskowski K, Stirling A, McKay WP, et al. A systematic review of intravenous ketamine for postoperative analgesia. Can J Anaesth 2011;58:911-23.

30. Perttunen K, Tasmuth T, Kalso E. Chronic pain after thoracic surgery: a follow-up study. Acta Anaesthesiol Scand 1999;43:563-7.

31 Pavy T, Medley C, Murphy DF. Effect of indomethacin on pain relief after thoracotomy. Br J Anaesth 1990;65:624-7.

32. Vadivelu N, Mitra S, Narayan D. Recent advances in postoperative pain management. Yale J Biol Med 2010;83:11-25.

33. Memis D, Inal MT, Kavalci G, et al. Intravenous paracetamol reduced the use of opioids, extubation time, and opioid-related adverse effects after major surgery in intensive care unit. J Crit Care 2010;25:458-62.

34. Khalili G, Janghorbani M, Saryazdi H, et al. Effect of preemptive and preventive ace- taminophen on postoperative pain score: a randomized, double-blind trial of patients under- going lower extremity surgery. J Clin Anesth 2013;25:188-92.

35. Apfel CC, Turan A, Souza K, et al. Intravenous paracetamol reduces postoperative nausea and vomiting: a systematic review and meta-analysis. Pain 2013;154:677-89.

36. Mac TB, Girard F, Chouinard P, et al. Acetaminophen Decreases Early Post-Thoracotomy Ipsilateral Shoulder Pain in Patients With Thoracic Epidural Analgesia: A Double-Blind Placebo-Controlled Study. J Cardiothorac Vasc Anesth 2005;19:475-8.

37. Bell RF, Dahl JB, Moore RA, et al. Perioperative ketamine for acute postoperative pain. Cochrane Database Syst Rev. 2006;(1):CD004603.

38. De Oliveira GS Jr, Castro-Alves LJ, Khan JH, et al. Perioperative systemic magnesium to minimize postoperative pain: ameta-analysis of randomized controlled trials. Anesthesiology 2013;119:178-90.

39. Waldron NH, Jones CA, Gan TJ, et al. Impact of perioperative dexamethasone on postoperative analgesia and side-effects: systematic review and meta-analysis. Br J Anaesth 2013;110:191-200. 
40. Cui W, Li Y, Li S, et al. Systemic administration of lidocaine reduces morphine requirements and postoperative pain of patients undergoing thoracic surgery after propofol-remifentanil-based anaesthesia. Eur J Anaesthesiol 2010;27:41-6.

41. Slovack M, Taylor B, Bryce R, et al. Does intravenous lidocaine infusion during video-assisted thoracoscopic surgery reduce postoperative analgesia? A randomized controlled study. Can J Anaesth 2015;62:676-7.

42. Hurley RW, Cohen SP, Williams KA, et al. The analgesic effects of perioperative gabapentin on postoperative pain: ameta-analysis. Reg Anesth Pain Med 2006;31:237-47.

43. Mishriky BM, Waldron NH, Habib AS. Impact of pregabalin on acute and persistent postoperative pain: a systematic review and meta-analysis. Br J Anaesth 2015;114:10-31.

44. Kinney MA, Mantilla CB, Carns PE, et al. Preoperative gabapentin for acute post-thoracotomy analgesia: a randomized, double-blinded, active placebo-controlled study. Pain Pract 2012;12:175-83.

45. Mishra A, Nar AS, Bawa A, et al. Pregabalin in Chronic Post-thoracotomy Pain. J Clin Diagn Res 2013;7:1659-61.

46. Yoshimura N, Iida H, Takenaka M, et al. Effect of Postoperative Administration of Pregabalin for Postthoracotomy Pain: A Randomized Study. J Cardiothorac Vasc Anesth 2015;29:1567-72.

47. Elmore B, Nguyen V, Blank R, et al. Pain Management Following Thoracic Surgery. Thorac Surg Clin 2015;25:393-409.

48. Loop T. Fast track in thoracic surgery and anaesthesia: update of concepts. Curr Opin Anaesthesiol 2016;29:20-5.

49. Yeung JH, Gates S, Naidu BV, et al. Paravertebral block versus thoracic epidural for patients undergoing thoracotomy. Cochrane Database Syst Rev. 2016;2:CD009121.

50. Kosiński S, Fryźlewicz E, Wiłkojć M, et al. Comparison of continuous epidural block and continuous paravertebral block in postoperative analgaesia after video-assisted thoracoscopic surgery lobectomy: a randomised, noninferiority trial. Anaesthesiol Intensive Ther 2016;48:280-7.

51. Kaplowitz J, Papadakos PJ. Acute pain management for video-assisted thoracoscopic surgery: an update. J Cardiothorac Vasc Anesth 2012;26:312-21.

52. Kotemane NC, Gopinath N, Vaja R. Analgesic techniques following thoraric surgery: a survey of United Kingdom practice. Eur J Anaesthesiol 2010;27:897-9.

53. Yamauchi Y, Isaka M, Ando K, et al. Continuous paravertebral block using a thoracoscopic catheter- insertion technique for postoperative pain after thoracotomy: a retrospective case-control study. J Cardiothorac Surg 2017;12:5.

54. Pace MM, Sharma B, Andreson-Dam J, et al. UltrasoundGuided Thoracic Paravertebral Blockade: A Retrospective Study of the Incidence of Complications. Anesth Analg 2016;122:1186-91.

55. Kelly ME, McNicholas D, Killen J, et al. Thoracic paravertebral blockade in breast surgery: Is pneumothorax an appreciable concern? A review of over 1000 cases. Breast J 2018;24:23-7.

56. Lekhak B, Bartley C, Conacher ID, et al. Total spinal anaesthesia in association with insertion of a paravertebral catheter. Br J Anaesth 2001;86:280-2.

57. Piccioni F, Colombo J, Fumagalli L, et al. Inadvertent high central neuroaxial block and possible total spinal anaesthesia occurring after nerve stimulation-guided thoracic paravertebral block. Anaesth Intensive Care 2014;42:270-1.

58. Foster LA, Deutz CK, Hutchins JL, et al. Total spinal and brainstem anesthesia as complication of paravertebral ropivacaine administration. Neurol Clin Pract 2017;7:430-2.

59. Voscopoulos C, Palaniappan D, Zeballos J, et al. The ultrasound-guided retrolaminar block. Can J Anaesth 2013;60:888-95.

60. Costache I, de Neumann L, Ramnanan CJ, et al. The midpoint transverse process to pleura (MTP) block: a new end-point for thoracic paravertebral block. Anaesthesia 2017;72:1230-6.

61. Kotzé A, Scally A, Howell S. Efficacy and safety of different techniques of paravertebral block for analgesia after thoracotomy: a systematic review and metaregression. Br J Anaesth 2009;103:626-36.

62. Detterbeck FC. Efficacy of methods of ontercostal nerve blockade for pain relief after thoracotomy. Ann Thorac Surg 2005;80:1550-9.

63. Mehran RJ, Martin LW, Baker CM, et al. Pain management in an enhanced recovery pathway after thoracic surgical procedures. Ann Thorac Surg 2016;102:e595-6.

64. Rice DC, Cata JP, Mena GE, et al. Posterior intercostal nerve block with liposomal bupivacaine: an alternative to thoracic epidural analgesia. Ann Thorac Surg 2015;99:1953-60.

65. Kent M, Alvelo-Rivera M, Luketich J. Minimally Invasive Surgery for Lung Cancer. In: Roth JA, Cox JD, Hong WK. Lung Cancer. Oxford: Blackwell Publishing, 
2008:180-93.

66. Blanco R, Parras T, McDonnell JG, et al. Serratus plane block: a novel ultrasound- guided thoracic wall nerve block. Anaesthesia 2013;68:1107-13.

67. Mayes J, Davison E, Panahi P, et al. An anatomical evaluation of the serratus anterior plane block. Anaesthesia 2016;71:1064-9.

68. Khalil AE, Abdallah NM, Bashandy GM, et al. Ultrasound-Guided Serratus Anterior Plane Block Versus Thoracic Epidural Analgesia for Thoracotomy Pain. J Cardiothorac Vasc Anesth 2017;31:152-8.

69. Ökmen K, Ökmen BM. The efficacy of serratus anterior block in analgesia for thoracotomy: a retrospective study. $\mathrm{J}$ Anesth 2017;31:579-85.

70. Okmen K, Okmen BM. Evaluation of serratus anterior plane block for pain treatment after Video-assisted thoracoscopic surgery. Anaesth Crit Care Pain Med 2017 pii: S2352-5568(17)30041-3.

71. Forero M, Adhikary S, Lopez H, et al. The erector spinae plane block: a novel analgesic technique in thoracic neuropathic pain. Reg Anesth Pain Med 2016;41:621-7.

72. Forero M, Rajarathinam M, Adhikary S, et al. Erector spinae plane (ESP) block in the management of post thoracotomy pain syndrome: a case series. Scand J Pain 2017. [Epud ahead of print].

Cite this article as: Piccioni F, Segat M, Falini S, Umari M, Putina O, Cavaliere L, Ragazzi R, Massullo D, Taurchini M, Del Naja C, Droghetti A. Enhanced recovery pathways in thoracic surgery from Italian VATS Group: perioperative analgesia protocols. J Thorac Dis 2018;10(Suppl 4):S555-S563. doi: $10.21037 /$ jtd.2017.12.86
73. Hamilton DL, Manickam B. Erector spinae block for pain relief in rib fractures. Br J Anaesth 2017;118:474-5.

74. Forero M, Rajarathinam M, Adhikary S, et al. Continuous erector spinae plane block for rescue analgesia in thoracotomy after epidural failure: a case report. A A Case Rep 2017;8:254-6.

75. Classification of chronic pain. Descriptions of chronic pain syndromes and definitions of pain terms. Prepared by the International Association for the Study of Pain, Subcommittee on Taxonomy. Pain Suppl 1986;3:S1-226.

76. Maguire MF, Ravenscroft A, Beggs D, et al. A questionnaire study investigating the preva- lence of the neuropathic component of chronic pain after thoracic surgery. Eur J Cardiothorac Surg 2006;29:800-5.

77. Rizk NP, Ghanie A, Hsu M, et al. A Prospective Trial Comparing Pain and Quality of Life Measures After Anatomic Lung Resection Using Thoracoscopy or Thoracotomy. Ann Thorac Surg 2014;98:1160-6.

78. Humble SR, Dalton AJ, Li L. A systematic review of therapeutic interventions to reduce acute and chronic post-surgical pain after amputation, thoracotomy or mastectomy. Eur J Pain 2015;19:451-65.

79. Kehlet H, Jensen TS, Woolf CJ. Persistent postsurgical pain: risk factors and prevention. Lancet 2006;367:1618-25. 